\title{
Linkage disequilibrium analysis of two genes mapping on OFC3: PVR and PVRL2
}

\author{
Furio Pezzetti ${ }^{1}$, Annalisa Palmieri ${ }^{1}$, Marcella Martinelli ${ }^{*}{ }^{1}$, Luca Scapoli ${ }^{1}$, Marzia Arlotti ${ }^{1}$, \\ Ugo Baciliero $^{2}$, Ernesto Padula ${ }^{2}$, Paolo Carinci ${ }^{1}$, Elisabetta Caramelli ${ }^{1}$ and \\ Francesco Carinci ${ }^{3}$
}

\author{
${ }^{1}$ Department of Histology, Embryology and Applied Biology, Centre of Molecular Genetics, University of Bologna, \\ Bologna, Italy; ${ }^{2}$ Department of Maxillo-Facial Surgery, San Bortolo Hospital, Vicenza, Italy; ${ }^{3}$ Department of \\ D.M.C.C.C., Chair of Maxillo-Facial Surgery, University of Ferrara, Ferrara, Italy
}

Clefts of the lip with or without cleft palate $(\mathrm{CL} / \mathrm{P})$ are one of the most common birth defects, occurring in 1/700-1/1000 infants born alive. The nature of the genetic contribution is still to be clarified; however, some chromosome regions and candidate genes have been proposed for this malformation. Recently, a couple of genes, PVR and PVRL2, mapping in the candidate region OFC3 on chromosome 19q13.31, have been investigated because of their homology to $P V R L 1$, a gene previously shown to cause the Margarita Island CL/P-ectodermal dysplasia syndrome. In the present work, we investigated PVR and PVRL2 genes by family-based linkage disequilibrium analysis using a sample collected from the Italian population. In contrast to previous analyses on other populations, we could not find any statistically significant association between the markers alleles and non-syndromic clefting.

European Journal of Human Genetics (2007) 15, 992-994; doi:10.1038/sj.ejhg.5201868; published online 30 May 2007

Keywords: orofacial cleft; PVR; PVRL2; OFC3

\section{Introduction}

Clefts of the lip and/or palate (CL/P) are a common craniofacial anomaly having a multifactorial aetiology with both genetic and environmental implications. The large number of candidate genes and loci that seem to be involved attest to the complexity of this malformation. ${ }^{1}$ One example of a clefting gene is the poliovirus receptor related-1 (PVRL1), which encodes nectin-1, a cell-to-cell adhesion molecule. Its loss of function was shown to cause the CL/P-ectodermal dysplasia (CLPED1; MIM 225060), a rare autosomal recessive syndrome. ${ }^{2}$

Recently, our group investigated $146 \mathrm{CL} / \mathrm{P}$ probands and 100 unaffected controls, ${ }^{3}$ all from an Italian population,

*Correspondence: Dr M Martinelli, Department of Histology, Embryology and Applied Biology, University of Bologna, Via Belmeloro 8, 40126 Bologna, Italy.

Tel: + 39051 2094101; Fax: + 39051 2094110;

E-mail: marcella.martinelli@unibo.it

Received 25 October 2006; revised 22 March 2007; accepted 3 May 2007; published online 30 May 2007 but failed to detect the common PVRL1 nonsense mutation $\mathrm{W} 185 \mathrm{X}$, which is seen as a genetic risk factor for nonsyndromic $\mathrm{CL} / \mathrm{P}$ in Northern Venezuela. ${ }^{4}$ In a further screening of the same sample, we found three rare sequence variants in PVRL1 exon $3 .^{5}$

Interestingly, two PVRL1-related genes, PVR and PVRL2, which alike encode nectin-related Ig-class cell-cell adhesion molecules that serve as viral receptors, both map in chromosome segment $19 \mathrm{q} 13.31-\mathrm{q} 13.32$, close to BCL3. Here is located OFC3, one of several non-syndromic CL/P susceptibility loci mapped in humans. ${ }^{6,7}$

We previously analysed the OFC3 candidate region using a sample of 40 multiplex pedigrees and microsatellite markers, and obtained evidence of linkage and linkage disequilibrium with APM and TDT analyses. ${ }^{8}$

Recently, Warrington et $a l^{9}$ searched for an association between orofacial clefts and mutations in the PVR and PVRL2 genes, using samples from different populations. They found a statistically significant association in the South American and Iowa populations for an allelic variant 
Table 1 SNPs information and TDT results

\begin{tabular}{lllcrr}
\hline dbSNP ID & Genome position $^{\mathrm{a}}$ & Gene position & Alleles $^{\mathrm{b}}$ & MAF $^{\mathrm{c}}$ & T/NT $^{\mathrm{d}}$ \\
\hline rs35385129 & chr19:49854029 & PVR-intron 6 & C/A & 0.13 & $59 / 42$ \\
rs1871047 & chr19:50043586 & PVRL2-intron1 & A/G & 0.45 & $92 / 82$ \\
\hline
\end{tabular}

aUCSC Genome Browser on Human May 2004 Assembly.

bSNP alleles in coding frame, major allele first.

'Minor allele frequency (MAF) calculated from all parental chromosomes.

${ }^{\mathrm{d}}$ Transmission/Non-Transmission (T/NT) counts are given for the major allele.

e $P$-value for TDT.

in the $P V R$ gene, while the $P V R$ marker did not show any significant association in the Danish sample.

\section{Materials and methods}

For the present work, we tested a sample of 230 affected patients and their parents, selected after informed consent from an Italian population, excluding syndromic cases. In order to classify the CL/P as non-syndromic and to exclude potential teratogenic influences, a careful anamnesis was carried out to evaluate the presence of any other somatic or neurological disorder in the family or the use of clefting substances, such as phenytoin, warfarin, and ethanol, during pregnancy. Eighty-five nuclear triads (fathermother-affected child) were enrolled, one for each multiplex pedigree, while 145 trios (father-mother-affected child) pertained to sporadic cases. DNA was extracted from peripheral blood samples as previously described. ${ }^{10}$

To investigate the linkage disequilibrium with $P V R$ gene, we used the pre-designed TaqMan assay C_1828143_10 for SNP having public ID rs35385129 at dbSNP at NCBI, while to test PVRL2 we chose the SNP assay C_1846421_10 (rs1871047). SNP analyses were performed using TaqMan SNPs genotyping assay (Applied Biosystems, Monza, Milan, Italy) run on an Applied Biosystems 7500 Sequence Detection System (Applied Biosystems, Monza, Milan, Italy). Linkage disequilibrium between SNP alleles and disease was tested with the transmission disequilibrium test (TDT), which examines the transmission of alleles from heterozygous parents to affected offspring. ${ }^{11}$

\section{Results}

Both markers employed were in Hardy-Weinberg equilibrium and none showed evidence of linkage disequilibrium. Table 1 summarises the information about the two markers used and the results of linkage disequilibrium analyses.

\section{Discussion}

Non-syndromic cleft lip with or without cleft palate is a complex genetic trait, and little is known about it.
Different chromosome regions and candidate genes have been proposed as causes in the aetiology of such congenital malformation. The OFC3 region has recently been further investigated for the presence of two genes, $P V R$ and PVRL2, because of their homology to PVRL1, a gene seen to be involved in a form of cleft. We investigated the two candidate genes for the presence of linkage disequilibrium, analysing the same two SNPs previously used by Warrington et al. ${ }^{9}$

In our investigation, no significant departure from random sharing was observed for both polymorphisms. A borderline over-transmission of the most frequent allele $\mathrm{C}$ $(P=0.09)$ at SNP rs35385129 was noticed, while the previous study showed significant overtransmission of the minor allele A. ${ }^{9}$ The investigation by Warrington et al was conducted with three different samples having comparable size with that used in the present study. The first from South America consisted of 434 patients and their mothers, the second from Iowa consisted of 205 affected/parents triads, and the third was composed by 147 triads from Denmark. Two populations, from South America and Iowa, showed significant linkage disequilibrium between oral clefts and PVR marker ( $P$-value 0.0007 and 0.0009 , respectively), while Danish population did not ( $P$-value 0.44$)$. In summary, our results partially confirm those reported by Warrington et al, since the analysis of Italian population did not show association between the $P V R$ polymorphism and the cleft, as observed for Danish. Altogether, data from both studies do not support $P V R$ involvement in CL/P onset among Europeans; however, it could not be excluded that one or more susceptibility alleles, not associated with rs35385129 alleles, might play a role in clefting. This evidence still reflects the complex aetiology of the CL/P malformation, which involves different genetic and environmental factors in distinct populations.

The results reported give additional strength to the fact that this disease presents genetic heterogeneity and in occasions allelic heterogeneity, which reflect the complex aetiology of CL/P. Therefore, ethnic and geographic variables are of paramount importance and must be considered if we are to have a precise definition of the onset of this congenital malformation. 


\section{Acknowledgements}

This study was supported in part by grants from Telethon E. 1147 (PC), MIUR local projects (FC, FP, and PC), CARIVERONA (FC, UB, $E P)$, Fondazione Cassa di Risparmio di Bologna (FP), and the Fondazione Cassa di Risparmio di Ferrara (FC).

\section{References}

1 Carinci F, Pezzetti F, Scapoli L et al: Recent developments in orofacial cleft genetics. J Craniomaxillofac Surg 2003; 14: 130-143.

2 Suzuki K, Hu D, Bustos T et al: Mutations of PVRL1, encoding a cell-cell adhesion molecule/herpesvirus receptor, in cleft lip/ palate-ectodermal dysplasia. Nat Genet 2000; 25: 427-430.

3 Scapoli L, Palmieri A, Pezzetti F et al: Investigation of the W185X nonsense mutation of PVRL1gene in Italian nonsyndromic cleft lip and palate patients. Am J Med Genet 2004; 127A: 211.

4 Sozen MA, Suzuki K, Tolarova MM, Bustos T, Fernandez Iglesias JE, Spritz RA: Mutation of PVRL1is associated with sporadic, non-syndromic cleft lip/palate in northern Venezuela. Nat Genet 2001; 29: $141-142$.
5 Scapoli L, Palmieri A, Martinelli M et al: Study of the PVRL1gene in Italian nonsyndromic cleft lip patients with or without cleft palate. Ann Hum Genet 2006; 70: 410-413.

6 Stein J, Mulliken JB, Stal S et al: Nonsyndromic cleft lip with or without cleft palate: evidence of linkage to $B C L 3$ in 17 multigenerational families. Am J Hum Genet 1995; 57: 257-272.

7 Wyszynski DF, Maestri N, McIntosh I et al: Evidence for an association between markers on chromosome $19 \mathrm{q}$ and nonsyndromic cleft lip with or without cleft palate in two groups of multiplex families. Hum Genet 1997; 99: 22-26.

8 Martinelli M, Scapoli L, Pezzetti F et al: Suggestive linkage between markers on chromosome 19q13.2 and nonsyndromic orofacial cleft malformation. Genomics 1998; 51: 177-181.

9 Warrington A, Vieira AR, Christensen K et al: Genetic evidence for the role of loci at 19q13 in cleft lip and palate. J Med Genet 2006; 43: e26.

10 Carinci F, Pezzetti F, Scapoli L et al: Nonsyndromic cleft lip and palate: evidence of linkage to a microsatellite marker on $6 \mathrm{p} 23$. Am J Hum Genet 1995; 56: 337-339.

11 Spielman RS, McGinnis RE, Ewens WJ: Transmission test for linkage disequilibrium: the insulin gene region and insulindependent diabetes mellitus (IDDM). Am J Hum Genet 1993; 52: 506-516. 